psychopraxis.neuropraxis 2015 · 18:153 DOI 10.1007/s00739-015-0282-x

Online publiziert: 7. September 2015 ๑) Springer-Verlag Wien 2015

CrossMark
Martin Aigner

Universitätsklinik für Psychiatrie und Psychotherapie, Abteilung für Erwachsenenpsychiatrie,

Universitätsklinikum Tulln, Tulln, Österreich

\title{
Stigmabefürchtungen in der Psychiatrie
}

\section{Sehr geehrte Leserinnen! Sehr geehrte Leser!}

Die Zeitschrift psychopraxis.neuropraxis bringt Falldarstellungen aus der Praxis. In dieser Ausgabe liegt der Schwerpunkt auf den „organischen psychischen Störungen“, die im ICD-10 im Kapitel F0 behandelt werden. Im ICD-10 finden wir sowohl ätiologisch als auch symptomatologisch definierte Kapitel. Das Kapitel F0 zählt zu den ätiologisch definierten Kapiteln, wo „organische, einschließlich symptomatische“"Faktoren als grundlegend für die Störungen angesehen werden. Dennoch werden nicht in allen Syndromdefinitionen organische Faktoren, insbesondere Biomarker, genannt. In der Ausbildung zum Facharzt für Neurologie bzw. Facharzt für Psychiatrie und psychotherapeutische Medizin ist diesem Spannungsfeld Rechnung getragen worden, indem durch ein sechsmonatiges Gegenfach, entweder Neurologie oder Psychiatrie, einmal mehr die organische Sichtweise und einmal mehr die syndromale Sichtweise erfahrbar wurde. Mit der neuen Ausbildungsordnung sind die Gegenfächer „verloren“ gegangen. Welche Auswirkungen diese Änderung auf die zukünftigen Fachärztinnen und Fachärzte hat, ist noch nicht abzuschätzen. Es besteht die Gefahr, dass durch das Fehlen von Gegenfächern die unterschiedlichen Herangehensweisen nicht mehr kennengelernt werden und dadurch der „breite Blick“ für die Praxis verloren geht. Durch die Basisausbildung ist es nicht systematisch gesichert, dass sowohl die neurologische als auch die psychiatrische Herangehensweise erfahrbar wird. Hier wird es eine Herausforderung sein, die Ausbildungsqualität zu halten. Eine Aufgabe, die beide Fächer nur gemeinsam bewältigen können!

Die neue Ausbildungsordnung bringt aus Sicht der Psychiatrie aber auch Verbesserungen in der Grundausbildung zum Allgemeinmediziner. Es hat sich die Erkenntnis durchgesetzt, dass psychische Störungen sehr häufig vorkommen und daher von Allgemeinmedizinern erkannt und auch therapiert werden sollten. Die Psychiatrie und psychotherapeutische Medizin ist zu einem dreimonatigen Pflichtmodul geworden. Fünf Schwerpunkte werden den Allgemeinmedizinern im Rahmen der Psychiatrie und psychotherapeutischen Medizin angeboten:

- Akut- und Notallmedizin,

- Basismedizin (20 Fälle),

- fachspezifische Medizin (30 Fälle),

- Gerontopsychiatrie (10 Fälle),

- Nachsorge der Patienten.

Die Sicherung der psychiatrischen Ausbildungsinhalte für andere Fächer ist besonders wichtig, da $60 \%$ der Erstverschreibungen von Psychopharmaka nicht von Psychiatern durchgeführt werden.

Stigmabefürchtungen sind immer noch stark, wenn es um den Kontakt mit der Psychiatrie geht. Obwohl „mental health" und „dental health " sich nur in einem Buchstaben unterscheiden, kann jeder ohne Stigmabefürchtungen in der Straßenbahn zur Begleitung sagen, dass er zum Zahnarzt fährt. Nicht so bei der Fahrt zum Psychiater. Die Integration der Psychiatrie in die Ausbildung zur Allgemeinmedizin kann diesem Stigma sicher entgegenwirken. Jedoch ist es auch wichtig, dass das Fach Psychiatrie von innen her mehr „stigmasensibel“ wird, und Diagnosen vermeidet, die Stigmabefürchtungen auslösen können. So der Begriff „Persönlichkeitsstörungen“. Dieser Begriff wird von vielen Patientinnen und Patienten als stigmatisierend erlebt, und es ist zu verstehen, dass niemand als ,gestörte Persönlichkeit“ etikettiert werden möchte. Eine Möglichkeit wäre es, diesen Begriff in "Interaktionsstörung" umzubenennen, da ja der zentrale Faktor dieser Störungsgruppe ein überdauerndes Muster an Interaktionsproblemen darstellt. Dies würde auch mehr auf eine Veränderbarkeit hindeuten und nicht dazu verleiten sich mit einer „Persönlichkeitsstörung“ zu identifizieren (z. B.: „Ich bin ein Bordie").

In diesem Sinne wünsche ich Ihnen eine gewinnbringende Lektüre der psychopraxis.neuropraxis für Ihre klinische Praxis.

\section{Martin Aigner}

\section{Korrespondenzadresse}

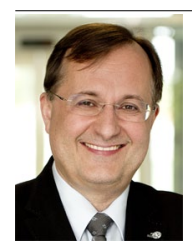

Prim. Priv.-Doz. Dr.

M. Aigner

Universitätsklinik für Psychiatrie und Psychotherapie, Abteilung für Erwachsenenpsychiatrie Universitätsklinikum Tulln Alter Ziegelweg 10, 3430 Tulln martin.aigner@tulln.lknoe.at 